\title{
SMALL SETS OF HOMEOMORPHISMS WHICH CONTROL MANIFOLDS
}

\author{
NORMAN LEVITT
}

\begin{abstract}
Let $N^{n}$ be a smooth connected paracompact manifold without boundary. A set $D$ of self-homeomorphisms of $M^{n}$ to itself is called controllable iff the semigroup generated by $D$ acts transitively on $M^{n}$.

THEOREM A. There is a complete vector field $X$ on $M^{n}$ and a selfhomeomorphism $H$ so that the set $D$ consisting of $H, H^{-1}$ and $X_{t}, t \in \mathbf{R}$, is controllable.

TheOREM B. Let $n \neq 4$ and let $M^{n}$ be compact and orientable. If $n$ is even, $>6$, let $M^{n}$ be simply connected. If $n \equiv 0(4)$, let signature $M^{n}=0$. Then there is a vector field $X$ on $M^{n}$ and a self-homeomorphism $H$ so that the set consisting of $\mathrm{H}, \mathrm{H}^{-1}$ and $X_{t}, t>0$, is controllable.
\end{abstract}

The purpose of this paper is to extend and modify some results of the author and H. Sussmann [5] concerning the controllability of a manifold by means of two vector fields. The reader is advised that [5] is an indispensable reference for the material below.

Let $M^{n}$ be a smooth, connected, paracompact manifold without boundary. Consider a set $D$ of homeomorphisms from $M^{n}$ to itself. For our purposes, we shall call $D$ controllable if and only if the semigroup of homeomorphisms generated by $D$ acts transitively on $M^{n}$. That is to say, $D$ is controllable provided that for any points $x, y \in M^{n}$ there is a homeomorphism $\Phi$ of $M^{n}$ to itself with $\Phi(x)=y$ and $\Phi$ of the form $\Phi=\phi_{1}^{n_{1}} \ldots \ldots \mid \phi_{j}^{n_{j}}$ where $n_{i} \geqslant 0$ and $\phi_{i} \in D$.

In the context of [5], controllability was discussed for sets of smooth complete vector fields $V^{j} \ldots V^{k}$. Let $V_{t}^{i}$ be the diffeomorphism which is obtained by applying the flow generated by $V^{i}$ for time $t$. Then, in the current terminology $V^{1}, \ldots, V^{k}$ is controllable if and only if the set $\left\{V_{t}^{i}, i=1\right.$, $2, \ldots, k, t \geqslant 0\}$ is controllable. The main result of [5] is that, given $M^{n}$, one can always find a pair of controllable vector fields $X, Y$, that is, the set $\left\{X_{t}\right.$, $t \geqslant 0\} \cup\left\{Y_{t}, t \geqslant 0\right\}$ will be controllable.

The aim of the present paper is to find a controllable set $D$ which is, in some sense, even "smaller" than that found in [5].

We state our results:

THEOREM A. Let $M^{n}$ be a connected paracompact smooth manifold without boundary. Then there is a smooth, complete vector field $X$ on $M^{n}$ and $a$ homeomorphism $H: M^{n} \rightarrow M^{n}$ so that the set $D$ consisting of $H, H^{-1}$ and all

Received by the editors September 29, 1975.

AMS (MOS) subject classifications (1970). Primary 57D25; Secondary 57D70.

Key words and phrases. Controllable set of vector fields, controllable set of homeomorphisms, gradient-like vector field, topologically conjugate vector fields, twisted double theorem. 
diffeomorphisms of the form $X_{t}, t \in \mathbf{R}$, is a controllable set.

In other words, Theorem A says that in the group of self-homeomorphisms of $M^{n}$, the subgroup generated by $D$ (which is the same as the semigroup generated by $D$, since $D$ is closed under taking of inverses) acts transitively on $M^{n}$. In fact, we actually obtain a slightly finer result.

THEOREM B. Let $M^{n}$ be a compact, connected orientable smooth manifold without boundary, $n \neq 4$. If $n \equiv 0$ (4), let the signature of $M^{n}$ be zero. If $n \geqslant 5$ let $M^{n}$ be simply connected.

Then there is a vector field $X$ on $M^{n}$ and a homeomorphism $H: M^{n} \rightarrow M^{n}$ so that the set $D$ consisting of $H, H^{-1}$ and all homeomorphisms of the form $X_{t}$, $t \geqslant 0$, is a controllable set.

(Recall that if $M^{n}$ is a closed oriented manifold of dimension $n \equiv 0$ (4), the signature is an invariant of oriented homotopy type defined as follows:

Since $n / 2$ is an even integer, the cohomology cup-product defines a symmetric bilinear pairing $H^{n / 2}\left(M^{n} ; \mathbf{R}\right) \otimes H^{n / 2}\left(M^{n} ; \mathbf{R}\right) \rightarrow \mathbf{R}$. The signature of this quadratic form is, by definition, the signature of the manifold. (See [3] for details.))

The proofs of Theorems A and B require a careful analysis of the proof of the main result of [5]. We therefore give a short outline of that proof. Recall that the purpose of [5] is to find a pair of controllable vector fields.

We start off in [5] with two vector fields having the following properties. $X^{0}$ is a gradient-like vector field for some proper Morse function $f$ on $M^{n}$ having a single relative and absolute minimum. (See [6], [7] for a good account of Morse functions.) This means $X^{0}(f) \geqslant 0$ and is equal to 0 only at the critical points of $f$ where $X^{0}$ also vanishes. $Y^{0}$ is the negative of $X^{0}$. In point of fact, however, all the techniques of [5] will go through if $Y^{0}$ is gradient-like for some Morse function having the same critical points as $f$ and if $Y^{0}=-X^{0}$ in a neighborhood of every critical point.

We then find a certain diffeomorphism $G: M^{n} \rightarrow M^{n}$ and let

$$
Y^{1}(x)=G_{*}\left(Y^{0}\left(G^{-1}(x)\right)\right)
$$

for $x \in M^{n}$. $G$ is a perturbation which is the identity except near the critical points. The effect of this change is simply to shift the critical points of $Y^{1}$ away from those of $X^{0}$ and to put "stable" and "unstable" manifolds for both flows in general position near critical points.

We emerge with a certain picture of the behavior of $X^{0}$ and $Y^{1}$ near the original minimum point $p$ of $f$. In particular, there is a disc $D^{n}$ surrounding $p$ with local coordinates $\left(x^{1}, \ldots, x^{n}\right)$. In these coordinates $X^{0}\left(x^{1}, \ldots, x^{n}\right)$ $=x^{1}\left(\partial / \partial x^{1}\right)+\cdots+x^{n}\left(\partial / \partial x^{n}\right)$, i.e., $X$ is the "outward pointing" vector field.

$$
f\left(x^{1} \cdots x^{n}\right)=\left(x^{1}\right)^{2}+\cdots+\left(x^{n}\right)^{2} .
$$

$Y^{1}$ is $-X^{0}$ near the boundary $S^{n-1}$ of $D^{n}$, but the flow lines converge to a single "sink" $q$ different from $p$.

We then construct a certain pair of vector fields, $A$ and $B$, which vanish outside a small collar neighborhood of $S^{n-1}$ in $D^{n}$. We set $X=X^{0}+A$, 
$Y=Y^{1}+B$. It then turns out that $X, Y$ is the desired controllable pair.

(For the reader who may wish to check the details of this construction in [5], it should be pointed out that, in the outline above, the notation is somewhat different from that used in [5]. The vector field $X^{0}$ above is also denoted $X^{0}$ in [5], but our $Y^{1}$ is the $Y^{0}$ of [5]. $A$ and $B$ are, respectively, denoted by $\phi(\zeta) \bar{X}(p)$ and $\phi(\zeta) \bar{Y}(p)$ in $[5, \S 7]$.)

We are now ready to prove Theorem $A$. Referring to the outline above, we assume that $X$ and $Y$ have been constructed starting from $X^{0}$ and $Y^{0}=$ $-X^{0}$.

Definition. Two vector fields $V$ and $W$ are said to be topologically (resp., smoothly) conjugate if and only if there is a homeomorphism (resp. diffeomorphism) $H$ so that $H \cdot V_{t}=W_{t} \cdot H$ for all $t \in \mathbf{R}$. $H$ is called the conjugating homeomorphism (resp. diffeomorphism).

Note that in the outline above, $Y^{1}$ is constructed so as to be smoothly conjugate to $Y^{0}$ with $G$ the conjugating diffeomorphism.

Furthermore, we claim that $X$, as constructed above, is topologically conjugate to $X^{0}$ and $Y$ is topologically conjugate to $Y^{1}$. The proof of this fact is as follows: In constructing $X, Y$, it turns out that in the disc, $D^{n}$, each trajectory of $X$ (except the degenerate trajectory which is the single point $p$ ) flows outward from $p$ and reaches the boundary $S^{n-1}$. (See $[5, \S 7]$.) That is, adding $A$ to $X^{0}$ to obtain $X$ introduces no new zeroes of the vector field and we still have $X(f) \geqslant 0$. (In fact, $A$ is constructed so that $X(f)=X^{0}(f)$.) Now let $G$ be defined as follows: Set $G=$ identity on the closure of $M^{n}-$ $D^{n}$. Let $G(p)=p$. Any point in $D^{n}-p$ is of the form $X_{t}^{0}(x)$ for some unique $s \in S^{n-1}, t \leqslant 0$. Similarly any point in $D^{n}-p$ is also of the form $X_{t}(s)$ in a unique way. We let $G\left(X_{t}^{0}(s)\right)=X_{t}(s)$.

Since this map is the identity on $S^{n-1}$ we obtain a well-defined map $G$ : $M^{n} \rightarrow M^{n}$ which is, in fact, a homeomorphism throwing the flow lines of $X^{0}$ onto the flow lines of $X$. (To check that $G$ and its inverse are continuous at $p$, note that $G$ sends $f^{-1}(\varepsilon)$ homeomorphically onto itself for $f(p) \leqslant \varepsilon$ $\leqslant f\left(S^{n-1}\right)$.) $G$ is thus the conjugating homeomorphism.

Similarly, $Y$ is conjugate to $Y^{1}$ by an identical construction (although checking continuity at $q$ is a bit less direct).

Now note that topological conjugacy is implied by smooth conjugacy and is an equivalence relation; moreover, if $V$ is topologically conjugate to $W$, then $-V$ is topologically conjugate to $-W$. Thus, $X$ is topologically conjugate to $-Y$ since in the sequence $X, X^{0},-Y^{0},-Y^{1},-Y$ each element is topologically conjugate to the next (recall we assume $Y^{0}=-X^{0}$ ).

Let $H$ be the conjugating homeomorphism, i.e.,

$$
H \cdot X_{t}=-Y_{t} \cdot H
$$

or equivalently

$$
H \cdot X_{t} \cdot H^{-1}=Y_{-t} \cdot
$$

The main result of [5] now assures us that, given $x, y \in M^{n}$, we may write

$$
y=X_{t_{1}} Y_{s_{1}} Y_{t_{2}} Y_{s_{2}} \cdots X_{t_{n}} Y_{s_{n}}(X)
$$

for some $t_{i}, s_{i} \geqslant 0$.

Applying $\left(1^{\prime}\right)$ we obtain 


$$
y=Y_{t_{1}} H X_{-s_{1}} H^{-1} X_{t_{2}} \cdots X_{t_{n}} H X_{-s_{n}} H^{-1}(X) .
$$

Thus, $X$ and $H$ play the role demanded in Theorem A and the proof of Theorem $\mathbf{A}$ is complete.

REMARK. Note that in order to move $x$ to $y$ we need only apply a homeomorphism which is a concatenation of $X_{t}$ 's, $H$ and $H^{-1}$, where between any two $X_{t}$ 's there occurs only $H$ or $H^{-1}$ and not any higher powers.

To prove Theorem B, we must use a few deeper results from differential topology. Assume, henceforth, that $\boldsymbol{M}^{n}$ satisfies the hypothesis of Theorem B, i.e., $M^{n}$ compact, connected, without boundary and orientable, $n \neq 4$, and if $n$ even, $>6, M^{n}$ simply connected, and signature $M^{n}=0$ if $n \equiv 0$ (4). We recall the "twisted double" theorem, which is known classically for 2 and 3 dimensional manifolds and which has been established for manifolds of dimension 5 and greater, under suitable restrictions, by a series of successively sharper results [10], [4], [11].

We quote the sharpest of these:

Proposition (WINKELNKEMPER [11]). ${ }^{1}$ Let $M^{n}$ satisfy the conditions above. Then $M^{n}$ may be decomposed as $M^{n}=W_{0}^{n} \cup W_{1}^{n}$ (where $W_{0}^{n}, W_{1}^{n}$ are connected codimensional-0 submanifolds with common boundary $\partial=\partial W_{0}^{n}$ $\left.=\partial W_{1}^{n}\right)$ such that $M^{n}$ admits a self-diffeomorphism $G: M^{n} \rightarrow M^{n}$ with $G\left(W_{0}^{n}\right)$ $=W_{1}^{n}, G\left(W_{1}^{n}\right)=W_{0}^{n}$.

We remark that for some bicollar neighborhood $\partial \times[-1,1]$ of $\partial, G$ : $\partial \times[-1,1] \rightarrow \partial \times[-1,1]$ and is given by $G(x, t)=(G(x),-t)$ for $x \in \partial$, $t \in[-1,1]$.

Since $W_{0}^{n}$ is connected we may find a Morse function $f: W_{0}^{n} \rightarrow[0, a]$ with a single relative and absolute minimum $p$ and with $\partial=f^{-1}(a)$ having no critical points. We extend $f$ to all of $M^{n}$ by setting $f(x)=2 a-f(G(x))$ for $x \in W_{1}^{n}$.

If $c$ is a critical point of $f$ recall (cf. [6], [7]) that there are "nice" local coordinates $\left(x_{c}^{1} \cdots x_{c}^{i}, y_{c}^{1} \cdots y_{c}^{n-i}\right)$ for a neighborhood around $c$ so that $c$ has coordinates $(0, \ldots, 0)$ and so that

$$
\begin{aligned}
& f\left(x_{c}^{1} \cdots x_{c}^{i}, y_{c}^{1} \cdots y_{c}^{n-i}\right) \\
& \quad=f(c)-\left(x_{c}^{1}\right)^{2}-\left(x_{c}^{2}\right)^{2}-\cdots-\left(x_{c}^{i}\right)^{2}+\left(y_{c}^{1}\right)^{2}+\cdots+\left(y_{c}^{n-1}\right)^{2}
\end{aligned}
$$

( $i$ is the index of the critical point).

Clearly, if $c \in W_{0}^{n}$ is a critical point of $f$, so is $d=G(c) \in W_{1}^{n}$ with index $c=n-$ index $d$. Moreover, we may obviously choose nice local coordinates for $c$ and $d$, respectively, so that

$$
x_{d}^{r}(G(x))=y_{c}^{r}(x), \quad y_{d}^{r}(G(x))=x_{c}^{r}(x)
$$

for $x$ in the coordinate patch around $c$.

We now modify $G$ to produce a new diffeomorphism $\bar{H}: M^{n} \rightarrow M^{n}$ which also interchanges $W_{0}^{1}$ and $W_{0}^{n}$. For each critical point $d \in W_{1}^{n}, d=G(c)$;

\footnotetext{
${ }^{1}$ We should point out that Winkelnkemper's result is stated for simply connected $M^{n}, n>6$. Barden's thesis [1] allows Winkelnkemper's method to be extended to the case where $n$ is odd, $>5, M^{n}$ non-simply-connected.
} 
thus critical points in $W_{1}^{n}$ correspond 1-1 to critical points in $W_{0}^{n}$. Write this correspondence as $d=d(c), c=c(d)$. Now $G$ will not, in general, throw nice coordinates around the critical point $d \in W_{1}^{n}$ onto nice coordinates around $c(d)$. In fact $G(c)$ is not necessarily $d(c)$. But we may apply the lemma of Cerf [2] and Palais [8] in order to isotop $G \mid W_{1}^{n}$ to a diffeomorphism $G_{1}$ : $W_{1}^{n} \rightarrow W_{0}^{n}$ so that:

(a) $G_{1}=G \mid W_{1}^{n}$ in a neighborhood of $\partial$,

(b) $G_{1}(d)=c(d)$ for all critical points $d$ of $f$ in $W_{1}^{n}$.

(c) For respective nice coordinates around $d$ and $c=c(d)$,

$$
x_{c}^{r}\left(G_{1}(x)\right)=y_{d}^{r}(x) ; \quad y_{c}^{r}\left(G_{1}(x)\right)=x_{d}^{r}(x) .
$$

We let

$\bar{H}$ has properties:

$$
\bar{H}= \begin{cases}G & \text { on } W_{0}^{n} \\ G_{1} & \text { on } W_{1}^{n}\end{cases}
$$

( $\alpha) \bar{H}$ permutes the critical points of $f$ taking critical points $c$ of index $i$ to critical points $d=d(c)$ of index $n-i$.

$(\beta)$ For nice coordinates around $c$ and $d$,

$$
x_{d}^{r}(\bar{H}(x))=y_{c}^{r}(x) ; \quad y_{d}^{r}(\bar{H}(x))=x_{c}^{r}(x) .
$$

Note that $(\alpha)$ and $(\beta)$ also hold when $\bar{H}$ is replaced by its inverse $\bar{H}^{-1}$.

Pick a Riemann metric for $M^{n}$ so that in a neighborhood of each critical point $c$ of $f$ with local coordinates $x_{c}^{1} \cdots x_{c}^{i}, y_{c}^{1} \cdots y_{c}^{n-i}$, the metric is the standard one with orthonormal basis $\partial / \partial x_{c}^{1} \cdots \partial / \partial x_{c}^{i}, \partial / \partial y_{c}^{1} \cdots \partial / \partial y_{c}^{n-i}$.

Let $X^{0}=\frac{1}{2} \cdot \operatorname{grad} f$ (with respect to this metric). Set $g=f \cdot \bar{H}^{-1}$ and let $Y^{0}$ be the vector field given by

$$
Y^{0}(x)=\bar{H}_{*}\left(X^{0}\left(\bar{H}^{-1}(x)\right)\right)
$$

Clearly, $Y^{0}$ is smoothly conjugate to $X^{0}$. We also have

$$
\begin{aligned}
Y^{0}(x)(g) & =\left[\bar{H}_{*} X^{0}\left(\bar{H}^{-1}(x)\right)\right]\left[f \cdot \bar{H}^{-1}\right] \\
& =\left[X^{0}\left(\bar{H}^{-1}(x)\right)\right]\left[f \cdot \bar{H}^{-1} \cdot \bar{H}\right]=\left[X^{0}\left(\bar{H}^{-1}(x)\right)\right](f) .
\end{aligned}
$$

Thus, $Y^{0}(x)(g) \geqslant 0$ for all $x$ and is 0 only if $\bar{H}^{-1}(x)$ is a critical point of $f$, i.e., $x$ is a critical point of $g$. Therefore $Y^{0}$ is a gradient-like vector field for the Morse function $g$. Now it is clear that critical points of $g$ of index $i$ are critical points of $f$ of index $n-i$ and that $Y^{0}=-X^{0}$ in a neighborhood of every critical point.

We may now apply the argument of [5], as outlined above, to produce a controllable pair of vector fields $X, Y$ with $X$ topologically conjugate to $X^{0}$ and $Y$ topologically conjugate to $Y^{0}$. Since topological conjugacy is an equivalence relation, $X$ is topologically conjugate to $Y$ by a homeomorphism $H$ so that $H \cdot X_{t} \cdot H^{-1}=Y_{t}$ for all $t$. For each $x, y \in M^{n}$ we have

$$
y=X_{t_{1}} Y_{s_{1}} \cdots X_{t_{n}} Y_{s_{n}}(x), \quad t_{i}, s_{i} \geqslant 0
$$

which we rewrite

$$
y=X_{t_{1}} H X_{s_{1}} H^{-1} \cdots X_{t_{n}} H X_{s_{n}} H^{-1}(x) .
$$


Thus, $X, H$ play the role demanded by Theorem $\mathrm{B}$ and the proof of Theorem B is complete.

REMARK. Note that to move $x$ to $y$ we need only apply a concatenation of $X_{t}$ 's, $t \geqslant 0, H$, and $H^{-1}$ where between any two $H_{t}$ 's there occurs only $H$ or $H^{-1}$, but no higher powers.

\section{REFERENCES}

1. D. Barden, The structure of manifolds, Thesis, Cambridge, 1963.

2. J. Cerf, Topologie de certains espaces plongements, Bull. Soc. Math. France 89 (1961), 227-380. MR 25 \#3543.

3. F. Hirzebruch, Topological methods in algebraic geometry, 3rd ed., Die Grundlehren der math. Wissenschaften, Band 131, Springer-Verlag, New York, 1966. MR 34 \#2573.

4. N. Levitt, Applications of engulfing, Thesis, Princeton University, 1967.

5. N. Levitt and H. Sussmann, On controllability by means of two vector fields, SIAM J. Control 13 (1975), 1271-1281.

6. J. W. Milnor, Morse theory, Ann. of Math. Studies, no. 51, Princeton Univ. Press, Princeton, N.J., 1963. MR 29 \#634.

7. , Lectures on the h-cobordism theorem, Princeton Univ. Press, Princeton, N.J., 1965. MR 32 \#352.

8. R. Palais, Extending diffeomorphisms, Proc. Amer. Math. Soc. 11 (1960), 274-277. MR 22 \#8515.

9. H. Seifert and W. Threlfall, Lehrbuch der Topologie, Teubner, Leipzig, 1934; reprint, Chelsea, New York, 1947.

10. S. Smale, On the structure of manifolds, Amer. J. Math. 84 (1962), 387-399. MR 27 \#2991.

11. H. E. Winkelnkemper, Thesis, Princeton University, 1970.

Department of Mathematics, Rutgers University, New Brunswick, New Jersey 08903 